\title{
Web-based Trainer for Electrical Circuit Analysis
}

Ludo Weyten, Pieter Rombouts, Jeroen De Maeyer

This document is an author's draft version submitted for publication to IEEE Transactions on Education. The actual version was published as:

L. Weyten, P. Rombouts and J. De Maeyer, "Web-Based Trainer for Electrical Circuit Analysis," IEEE Trans. Education, Vol. 52, No. 1, pp. 185-189, Feb. 2009. 


\title{
Web-based Trainer for Electrical Circuit Analysis
}

\author{
Ludo Weyten, Member, IEEE, Pieter Rombouts, Member, IEEE, \\ Jeroen De Maeyer Member, IEEE
}

\begin{abstract}
A web-based system for training electric circuit analysis is presented in this paper. It is centered on symbolic analysis techniques and not only it verifies the student's final answer but it also tracks and coaches $\mathrm{him} / \mathrm{her}$ through all steps of his/her reasoning path. The system mimics homework assignments, enhanced by immediate personalized feedback. Evaluation data based upon a first trial indicate that this tool is a useful complement to the traditional training approach.
\end{abstract}

Index Terms-e-learning, circuit analysis, analog electronics, web-based tutoring, personal coaching

\section{INTRODUCTION}

Circuit analysis is one of the cornerstones of electrical engineering. When first learning circuit analysis, students should be familiarized with fundamental concepts through the use of simple examples. From this basis, substantial training will prepare them to analyze real-world circuits [1]. Since computer-based simulation tools such as SPICE and QUCS are readily available [2], it is common practice to introduce the students to the powerful features of these programs. These tools enhance the learning process if they enable the students to explain or even anticipate the simulation results [3]; otherwise, their use merely becomes a mechanical exercise.

A symbolic approach, where relevant quantities are manipulated as symbols without assigning them specific numerical values, is a more powerfull tool for developing insight into a circuit's behavior [1]. Symbolic network analysis is useful for optimizing circuit performance or for selecting suitable circuit configurations to realize given specifications. Symbolic techniques are therefore considered to be essential tools in the design process.

Manuscript received Dec 17, 2006; revised Nov 15, 2007.

L. Weyten and P. Rombouts are with the Electronics and Information Systems Laborotory (ELIS) at the Engineering Faculty of Ghent University, Belgium. When this work was done, Jeroen De Maeyer was with the Electronics and Information Systems Laborotory (ELIS) at the Engineering Faculty of Ghent University, Belgium. He is now with AMIS BVBA an ON Semiconductor company, Oudenaarde, Belgium. .
The algebraic manipulations involved may of course be carried out by hand, but once the problem has been translated into a a set of algebraic relations, symbolic mathematical tools (Maple, Maxima, Derive,...) may be used to process and simplify equations and to present the results [3], [4]. Another step in automating certain parts of the analysis is made possible by the availability in recent years of symbolic simulation packages [5], where algebraic expressions are derived directly from the circuit's schematic.

Whatever the degree of automation used in the actual design process, students are expected to acquire a thorough understanding of circuit properties. This understanding is typically begun by learning fundamental principles and studying some basic circuits (both analytically and through numeric simulations). In a second learning stage, the students are expected to develop more comprehensive reasoning skills, based upon known circuit properties and principles. In particular they should be able to predict certain results by mere inspection of the schematic, without even starting the analysis; so for example they should notice that a capacitor in parallel with a voltage source has no influence whatsoever on other voltages in the network. As another example, invoking Thévenin's theorem, they might notice that certain resistors make up a parallel connection and appear as such in all transfer functions. Merely by inspecting the schematic, the students should know the order of the transfer function. Some more typical examples of this type of reasoning are to be found in [1].

The extent to which these skills should be developed depends on the student's educational track: for students oriented toward digital system design, a minimum level is needed, whereas for students specializing in analog circuit design far more experience is required.

In an introductory circuits course for systemoriented students, training in more comprehensive reasoning should be introducesd. This training 
should be further developed in follow-up (specialist) courses on analog electronics where students are expected to develop a more sophisticated circuitbased reasoning. These learning objectives can be achieved by exposing students to a set of suitable problems (homework). Commonly, the solutions are made available after completion of an exercise. In order to verify partial results or to find out where (and why) their reasoning went wrong, students may contact an instructor individually. This process often turns out to be a very time-consuming activity for tutors. In order to provide adequate feedback to the students, the web-based tool presented in this paper was developed as a means of personalized training and self-evaluation, and to help students to bridge the gap between an introductory circuit course and the follow-up courses. The tool was used at the Engineering Faculty of Ghent University, Belgium, in the Analog Electronics course in 2005-2006 and in 2006-2007 as a complement to the regular classes and as a partial substitute for homework assignments.

In Section II, the pedagogical approach behind the Web-based trainer is explained in detail. Section III deals with the specific interface with the student that was developed. In Section IV the web engine behind this interface is explained. In Section V, results of an actual test are discussed as well as the students' appraisal of the tool. Finally in Section VI conclusions are presented.

\section{Pedagogical approach}

The basis of the approach is taken to mimic as far as possible the ideal situation where a student is solving a problem by herself or himself but where a tutor is available on call. Any action should be initiated by the student, and after each assertion she or he makes, its validity is either approved or denied by the system, playing the role of the tutor. In order to avoid guesswork from the student, a record is kept of her or his actions. Sometimes the student is asked to give further justification for her or his statement, much as a tutor might if the information provided by the student is not adequate to proceed. After completion of the exercise, the student gets a final rating of the correctness of her or his reasoning and an appraisal of the extent to which this was justified when asked.

This pedagogical approach can be demonstrated through an example. The student is given the

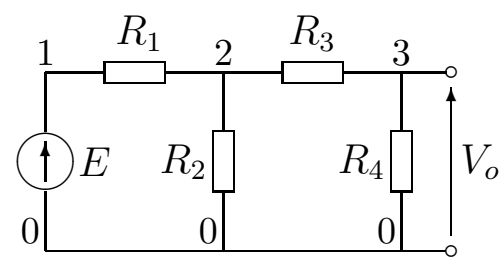

Fig. 1. Initial schematic for problem 1

schematic shown in Fig. 1. and is asked to derive an expression for the output voltage $V_{o}$ as a function of the source value $E$ and the network elements. An inexperienced student might solve this problem by writing down Kirchhoff's current law at nodes 2 and 3 and solving the resulting set of equations.

This typical straightforward solution may be desirable in a first course on networks, but in a followup course such a standardized solution is often discouraged because it does not incite the student towards creative thinking. Preferably, the student should use her or his knowledge of previously studied circuits to gain more insight in this situation and to arrive at a solution faster without performing extensive calculations.

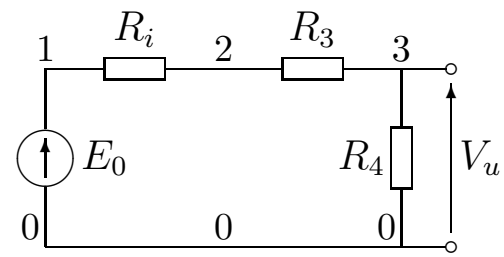

Fig. 2. Simplified schematic obtained while solving problem 1

A more desirable response might go as follows. The student might notice that the sub-circuit $\{E$, $\left.R_{1}, R_{2}\right\}$ can be replaced by a simpler equivalent consisting of a voltage source connected in series with a single resistor using Thévenin's theorem. By this means, the situation is reduced to the schematic represented in Fig. 2. Now the student should recognize a voltage divider, and use her or his knowledge of this previously studied circuit. If the student envisages the basic voltage divider circuit as consisting only of two resistors, she or he might need some intermediate steps in her or his reasoning; namely replacing the series connection of $R_{i}$ and $R_{3}$ by a single resistor and applying the well-known formula

$$
R_{S}=R_{i}+R_{3}
$$

for its value. In either case, by inspection of the 
network the student comes close to the final solution: she or he merely has to write down the voltage divider formula

$$
V_{o}=\frac{R_{4}}{R_{i}+R_{3}+R_{4}} E_{o}
$$

and the expression for the internal resistance

$$
R_{i}=\frac{R_{1} R_{2}}{R_{1}+R_{2}}
$$

from Thévenin's theorem.

Analyzing this typical solution delivered by a well-behaved student, it can be broken up into a sequence of statements falling into two categories. Firstly, there are statements about recognizing a subcircuit or applying a network property, and secondly there are algebraic formulae that relate an unknown quantity to other (symbolic) quantities in symbolic expressions.

The student could invoke some of these statements in a different order, so for example while invoking Thévenin's theorem, she or he might be aware that she or he would need expression (3) and thus formulate it earlier in the reasoning chain. So the order of some statements could be interchanged, but at the end of the solution process, all of the relevant statements should have been made. Of course, some statements must precede other statements (prerequisites).

With this model of the student's behavior in mind, the procedure was automated as will be described in Section III. The approach differs from that of related work in this field. Research groups at several universities have developed advanced systems for course delivery [6]-[8] including automated homework pages with on-line grading. To the authors' knowledge, these systems give tutors the means to formulate homework assignments with different sets of numerical values [7], [8], and enable the students to check their final (numerical) results by filling in an appropriate form. The system presented in this paper is centered on symbolic methods, rather then numeric ones which must be mastered by future circuit designers. Furthermore, this system will track or coach the student through all steps of her or his reasoning path, rather than just approving the final outcome. This capability allows the reasoning of the student to be verified, and when necessary guided by direct 'individual' feedback from the system.

\section{WEB INTERFACE}

From the observations in the previous paragraph it is obvious that two types of statements can be expected from the student: firstly to recognize schematic-related situations and secondly to write down mathematical expressions. In the new webbased system, the first type of statement entry is implemented by presenting a series of possible theorems and/or sub-circuits and inviting the student to make a choice among this set (a free response is not possible here). As soon as she or he made an appropriate choice, the system will ask her or him to indicate the relevant sub-network of the problem's schematic by clicking on its individual circuit elements. If the student does this correctly, the system acknowledges this, and possibly presents a new schematic, modified according to the circuit property or theorem that was invoked. For instance, applying Thévenin's theorem will simplify the network. So if the student clicks on the elements $E, R_{1}$ and $R_{2}$ and states she or he wants to use Thévenin's theorem, the interface will simplify the network of Fig. 1. to that of Fig. 2 as shown in Fig.3.

As for statements consisting of an algebraic expression, these could also be made through textual input fields in a form. In practice, however, this method of entry turned out to be quite sensitive to typing errors or misinterpretations (in particular with subscripts). Therefore another approach was introduced: all the symbols making up a formula can be selected by clicking on the corresponding network elements. This guarantees that the student exclusively uses symbols already present in the schematics shown on the screen. Additional symbols such as,+- or $\omega$ are provided by means of a small menu.

This alternative method has an important advantage from a pedagogical viewpoint: the student makes a strong visual association between entities in the formula and the corresponding circuit elements in the schematic (thereby bypassing in the student's mind the link through a particular name).

Each successful step that brings the student closer to the solution is summarized permanently on the screen. Just as in a pencil and paper solution, the whole summary is presented as a list of one-line statements that paraphrase the student's statements, as shown in Fig.3.

As the student progresses towards the solution 


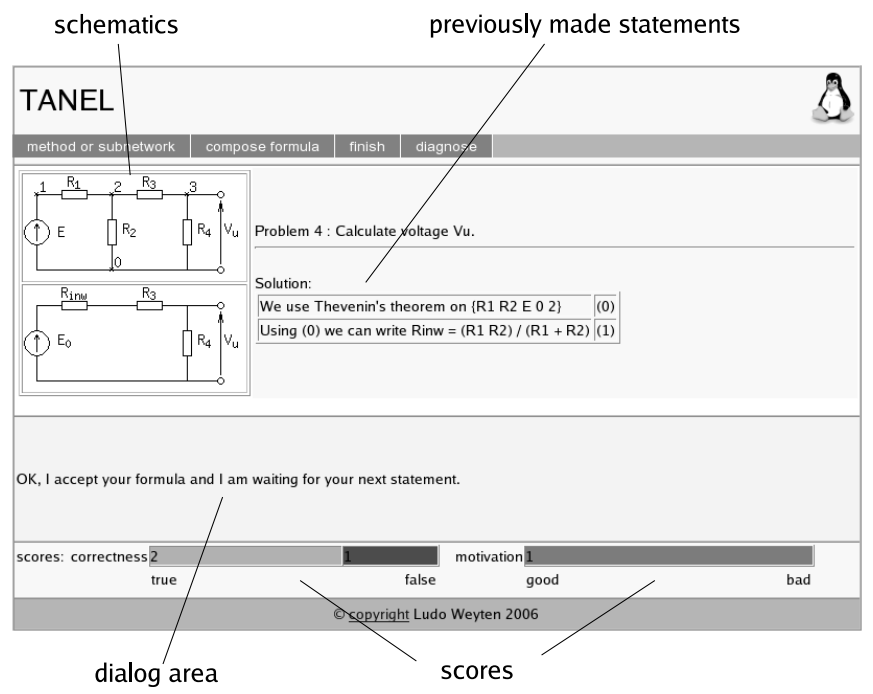

Fig. 3. Screen shot of the web-interface (English version)

of the problem, the system keeps several scores. One of these is the ratio of the number of correct statements that contribute to solving the problem, relative to the total number of statements. Another is the percentage of correctly substantiated statements.

\section{DATA PROCESSING AND FEEDBACK GENERATION}

Behind the web interface is a PHP- and MySQLdriven engine at the server side. As mentioned above, a set of theorems and/or sub-circuits is presented to the student, drawn from a database containing data on network theorems and sub-circuits such as Thévenin's theorem, the voltage divider, etc. In view of software localization, these data are multilingual.

Next, the student has to identify the relevant subnetwork by selecting the elements it contains. Such a list is of course problem-specific. In order to validate this type of student response, an appropriate data set is needed for each problem. These data are stored in the problem-specific database. When the student chooses to make a formula-type statement, the input data are also checked against possible correct statements stored in the problem-specific database.

Before actually comparing the data, a normalizing operation is necessary, otherwise some perfectly legitimate variations would not be allowed. Simply stated, the system should accept either $R_{S}=R_{i}+R_{3}$ or $R_{S}=R_{3}+R_{i}$ for Eq. (1). In order to accomplish this, the numerator and denominator of algebraic expressions are expanded as polynomials and their terms are lexicographically ordered. Both the reference data and the student's reply are processed by the same standardizing algorithm. Lexicographical ordering is also used for the standardization of element lists.

Populating the problem-specific database is of course a task for the tutor. Gathering and formulating this type of problem-specific information is not as difficult as it may seem. The approach taken here, is to provide a special tutor mode: in this mode, the system accepts all statements made by the user without verification. These statements are assumed to be correct and relevant to the solution of the problem and as such are registered in its specific data set. In this phase, the standardizing algorithm is applied to the input data in order to prepare them for later use in the verification of the correctness of the student's statements. The tutor populates the data set simply by solving the problem using the student's standard interface. Of course, several different solutions may be introduced.

In addition to the theorem and sub-circuit database and the problem-specific database, a third database is provided for logging purposes. In this database, all student statements, correct as well as incorrect, are registered and time-stamped. This information enables the instructor to follow each student's progress for each problem. It is also possible to analyze all the data relative to a particular problem for the whole group of students, in order to identify frequently occurring mistakes, or particular preferences in solving particular problems.

\section{Evaluation}

The web-based tool was introduced in the Engineering Faculty of Ghent University in the third year of the Bachelor's degree in Electrical Engineering, during the academic year 2005-2006. The tool was used on a voluntary basis by 26 students from a class of 49. During the academic year 2006-2007 its use was part of the compulsory problem solving sessions, in a class of 33 students.

\section{A. Student Performance}

Student activity was monitored and appropriate reports were generated from the system's database. Each combination of a student and an exercise begun by that student was considered. In 2005-2006, 
$49 \%$ of these combinations resulted in a successful solution. From the remaining $51 \%$ a further $21 \%$ were partly solved without the student having made any mistake. Since the students participated on a voluntary basis, a potential interpretation of these incomplete solutions is that the student was not stuck, but simply did not wish to finish this exercise. In the remaining $30 \%$, the student made some mistakes and did not solve the problem.

Since there was no stringent incentive for the student to finish solving the problem, the fraction of successfully completed exercises might not be suitable as a performance indicator. Therefore, another criterion should be used to measure the quality of the students response. So, the set of all student statements was considered. The percentage of correct statements was $71 \%$. For the correct statements where a further justification was required from the student, some $72 \%$ were correctly substantiated. These indicators can also be calculated for any particular student.
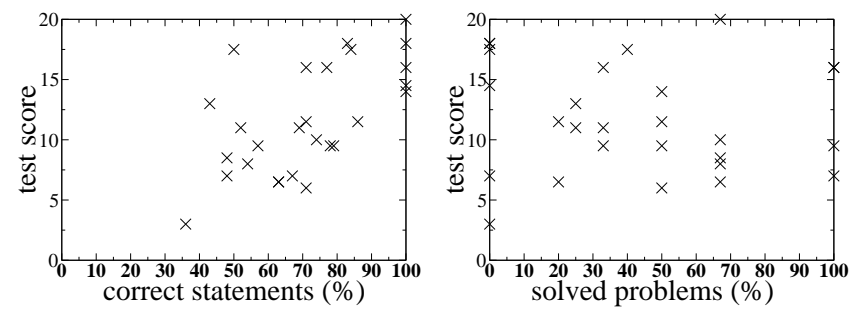

Fig. 4. Test score vs. fraction of correct statements (left) and test score vs. solved problems (right)

In order to verify the plausibility of the student's individual performance indicators, their correlation with the scores obtained in a classical written test was calculated. The test was taken at the end of the period in which the web-based tool was used, but it covered a broader set of circuit problems. Nevertheless, as shown in Fig.4. (left), a significant correlation (Pearson 0.61) was found between the scores obtained in the classical test and the fraction of correct statements. If one outlier $(50 \%, 17.5)$ is eliminated, the Pearson correlation is 0.69 . No significant correlation was found with the fraction of successfully completed exercises (see fig. 4, right). This result can be explained by the fact that students were not obliged to entirely finish the partly solved exercises.

The average scores out of 20 in the classical written test were 11.7 (standard deviation 4.5) for the group of students using the web-based trainer and 10.3 (standard deviation 4.6) for the control group who did not use it. In order to compare the distributions of both groups of samples, a (nonparametric) Mann-Whitney test was used. Here a normalized deviation of $0.71 \sigma$ was found, indicating that the null hypothesis (i.e., the probability distribution of both populations being equal) is $24 \%$. Although there might be a difference in performance between both groups, it is hardly significant. As mentioned above, the written test covered a broader set of circuit problems than the web-based trainer, which may explain the weak effect on the students' performance. At this stage, the main purpose of the trainer was to reduce the tutor's workload. It should be noted that this reduction of the workload has not yet been realized because the trainer was offered as an additional resource to the students and did not replace the traditional exercise sessions.

In 2006-2007, using the trainer was part of the compulsory problem solving sessions, meaning that there was no control group. The students solved $70 \%$ of the problems and the fraction of correct statements was $72 \%$.

\section{B. Assessment by Students}

After the conclusion of the semester and the exam, the students were asked to give feedback on their experience with the system, both in 2005 2006 and in 2006-2007. In order to encourage an unreserved response, the survey was organized anonymously. A total of 23 participants filled out the questionnaire. The results are presented in Table I.

The questionnaire was structured with answers on a five point Likert-like scale (strongly disagree / disagree / neutral / agree / strongly agree). Moreover, students were able to make any remarks they might consider to be useful (free text).

Most respondents considered the system to be useful. They agreed that is was a nice complement to the traditional homework but they disagreed that it would be a replacement, an aspect which was not targeted in the first place. The students generally appreciated the user interface, but specific feedback was given that may lead to an improved version of the software.

From the respondents' free remarks as well as from other (informal) interviews with users of the 


\begin{tabular}{|l|c|c|c|c|c|}
\hline statement about & \multicolumn{2}{|c|}{ disagree } & neutral & \multicolumn{2}{c|}{ agree } \\
the application & -- & - & 0 & + & ++ \\
\hline I used it several times & 4 & 2 & 0 & 14 & 3 \\
It is a useful tool & 1 & 1 & 4 & 15 & 2 \\
It is user-friendly & 2 & 5 & 5 & 11 & 0 \\
The interface is good-looking & 0 & 0 & 4 & 16 & 3 \\
The direct feedback is useful & 0 & 4 & 6 & 10 & 3 \\
It replaces homework & 2 & 10 & 5 & 4 & 2 \\
It complements homework & 0 & 3 & 2 & 13 & 5 \\
\hline
\end{tabular}

TABLE I

ASSESSMENT BY STUDENTS

system it became clear that being under the constraint (imposed by the system) of developing a strictly sound and well-motivated reasoning was displeasing. Nevertheless, the purpose of this system is precisely that, to stimulate accurate and clearcut reasoning about circuits. In the authors' opinion, this feeling might be linked to the noncommittal and entertaining nature of web-surfing. The expectations of the user are at odds with the the rigor and precision that is typical for scientific and technical reasoning. As web-based course delivery and e-learning systems are becoming more and more commonplace, this discord is expected to vanish.

\section{CONCLUSION}

In this paper a new web-based system for training students in the framework of teaching electric circuit theory and electronics was presented. The novelty of the approach is that it is centered on symbolic analysis techniques which are essential in a design context. Furthermore, the system tracks and coaches the student through all the steps of her or his reasoning path rather than just approving or disapproving the final outcome.

The main benefit is that the system approximates a well-established method of providing practical (homework) assignments enhanced by immediate individualized feedback provided by a private tutor. Since student reasoning is tracked very closely, the system can be used to obtain valuable information for the tutor about possible improvements in her or his teaching.

The system was introduced in the regular curriculum. The students performance has been discussed as well as the results of a survey traken of the users. Both indicate that the new tool could be a valuable complement to the traditional homework assignments.
The approach presented here might also be extended to other subjects where similar reasoning structures based upon schematic representations and algebraic expressions are to be found. Other applications, such as analytic geometry, may be options for further development. At present, suitable tools are being developed to facilitate the introduction of new assignments and to follow the student performance.

\section{REFERENCES}

[1] L. Huelsman, "Symbolic analysis - a tool for teaching undergraduate circuit theory," IEEE Transactions on Education, vol. 39, no. 2, pp. 243-250, May 1996.

[2] D. O. Pederson, "A historical review of circuit simulation," IEEE Trans. on Circuits and Systems, vol. 31, no. 1, pp. 103-111, Jan. 1984.

[3] H. Pota, "Computer-aided analog electronics teaching," IEEE Transactions on Education, vol. 40, no. 1, pp. 22-35, February 1997.

[4] J. Teng, J. Fidler, and Y. Sun, "Symbolic circuit analysis using mathematica," International Journal of Electrical Engineering Education, vol. 31, no. 4, pp. 324-332, 1994.

[5] A. Luchetta, S. Manetti, and A. Reatti, "Sapwin - a symbolic simulator as a support in electrical engineering education," IEEE Transactions on Education, vol. 44, on CD accompanying no. 2, May 2001.

[6] B. P. Butz, M. Duarte, and S. M. Miller, "An intelligent tutoring system for circuit analysis," IEEE Transactions on Education, vol. 49, no. 2, pp. 216-223, May 2006.

[7] L. Palma, R. F. Morisson, P. N. Enjeti, and J. W. Howze, "Use of web-based materials to teach electric circuit theory," IEEE Transactions on Education, vol. 48, no. 4, pp. 729-734, November 2005.

[8] C. R. Smaill, "The implementation and evaluation of oasis: A web-based learning and assessment tool for large classes," IEEE Transactions on Education, vol. 48, no. 4, pp. 658-663, Nov. 2005.

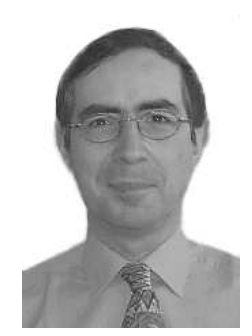

Ludo Weyten Ludo Weyten was born in Mortsel (Antwerpen, Belgium) in 1947. He received the Ir. degree and the Dr. degree from Ghent University (Belgium) both in Electrical Engineering, in 1970 and 1978 respectively. From 1970 to 1972 he joined the Electronics Laboratory of the same university as a research assistent. From 1972 to 1975 he was teaching at the National University of Zaire (now Congo) at Lubumbashi in a government technical co-operation project. Since 1975 he is with the Engineering Faculty of Ghent University (Belgium) where he is currently professor. His teaching and research interests are in the field of electronics circuits and systems and e-learning. 


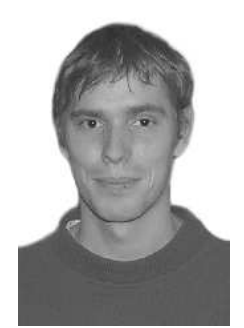

Pieter Rombouts Pieter Rombouts was born in Leuven, Belgium in 1971. He obtained the Ir. degree in applied physics and the Dr. degree in electronics from Ghent University in 1994 and 2000 respectively. Since 1994 he has been with the Electronics and Information Systems Department of the Gent university where he is currently professor. In 2002 he was a visiting professor at the University Carlos III in Madrid (Spain). His technical interests are signal processing, circuits and systems theory and analog circuit design.

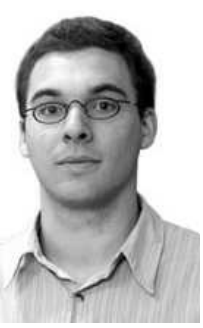

Jeroen De Maeyer Jeroen De Maeyer was born in Brugge, Belgium in 1979. He obtained the Ir. degree in electronics in 2002 from Ghent University, Belgium. Upon graduation he joined the Electronics and Information Systems Department of the same university where he obtained his $\mathrm{PhD}$ in 2006 with the support of the Science Foundation (F.W.O.Vlaanderen). His research focusses on A/D and D/A conversion, more specifically continuous time sigma delta modulators. Currently he works as a design engineer/project leader at AMIS BVBA part of ON Semiconductor in Oudenaarde, Belgium. 